\title{
DISPONIBILIDAD LÉXICA DE HABLANTES EXTRANJEROS ESTUDIANTES DE ESPAÑOL COMO SEGUNDA LENGUA EN SANTIAGO DE CHILE: ESTUDIO PRELIMINAR DE LAS DIFERENCIAS DE SEXO
}

\author{
Lexical availability of foreign speakers students of Spanish as a second language in \\ Santiago de Chile: preliminary study of sex differences
}

\section{Ángela Galdames Jiménez*}

Silvana Guerrero González ${ }^{\dagger}$

Gloria Toledo Vegat

\begin{abstract}
RESUMEN
En esta investigación, se analizan las diferencias de sexo en la disponibilidad léxica escrita de los hablantes no nativos de español como segunda lengua en etapa inicial de inmersión, en Santiago de Chile. Con base en los discursos escritos de 17 hombres y 17 mujeres, se identifica el léxico disponible, se cuantifica y se correlaciona su disponibilidad con la variable sexo. Asimismo, se intenta determinar el léxico disponible común a la variable externa sexo con el propósito de facilitar y guiar la adquisición por nivel en los cursos de E/LE. Con este estudio, se intenta comprobar dos hipótesis: por un lado, que hay diferencias en el manejo de léxico disponible escrito entre hombres y mujeres estudiantes de español como segunda lengua y, por otro, que se puede determinar un léxico común a ambos sexos al inicio de la inmersión en Santiago de Chile. El análisis de los datos permite concluir que mientras las mujeres presentan una mayor disponibilidad léxica, los hombres registran una menor disponibilidad con tendencia a la repetición del léxico disponible que utilizan. Además, se comprueba que es factible determinar un léxico común a ambos sexos.
\end{abstract}

Palabras clave: sociolingüística, variación léxica, léxico disponible, español como segunda lengua.

\begin{abstract}
This investigation analyzes differences in sex, regarding to the written lexical availability in non-native speakers of Spanish as second language who are in an initial period of immersion in Santiago, Chile. This study is based on 34 written texts (17 of men and 17 of women), and identifies the common written available lexicon between both sexes to facilitates and guide the acquisition in SSL classes in every level. With this study, we attempt to prove two hypotheses: on one hand, that there are differences in the written available lexicon between men and women who study SSL, and on the other hand, that a common lexicon can be determined between sexes at the beginning of the immersion process in Santiago, Chile. The data analysis allows to conclude that while women show greater lexical availability, men register a minor one, and tend to repeat the available words they use. Likewise, we checked that is feasible to determine a common lexicon between both sexes.
\end{abstract}

Key Words: Sociolinguistics, lexical variation, lexical availablity, Spanish as second language.

${ }^{*}$ Lic. Pontificia Universidad Católica de Chile. Chile

Correo electrónico: agaldame@uc.cl

${ }^{\dagger}$ Dra. Universidad de Chile. Chile.

Correo electrónico: siguerrero@u.uchile.cl

${ }^{\ddagger}$ Dra. Pontificia Universidad Católica de Chile. Chile.

Correo electrónico: gtoledo@uc.cl

Recepción: 17-05-2017_Aceptación: 02-02-2018 


\section{Introducción}

El estudio del español en el mundo, como segunda lengua o lengua extranjera, ${ }^{\S}$ se ha convertido en una herramienta necesaria y eficaz. Por una parte, para tener mayor acceso al conocimiento laboral y académico y, por otra, como un mecanismo de acercamiento al mundo hispano y su cultura. Según el Instituto Cervantes, el español es la segunda lengua más hablada en el mundo y existen más de 548 millones de personas que la utilizan, de las cuales casi 470 millones la tienen como lengua materna, mientras la cifra restante incluye a los cerca de 20 millones de personas que la estudian como lengua extranjera y a los que tienen una competencia limitada de ella. El Instituto Cervantes señala, además, que “en 2030 los hispanohablantes serán el 7,5\% de la población mundial” (Centro Virtual Cervantes, 2015, p. 5). Respecto de los números y estadísticas, se puede ver la relevancia que tiene el estudio del español como lengua extranjera (E/LE) y, por ende, la necesidad de su enseñanza, tanto en aula de clases, como en contextos de inmersión no formales en países de habla hispana. Por esta razón, realizar estudios de las diversas ramas de lingüística aplicada a E/LE puede ayudar a dilucidar dificultades y necesidades en la adquisición del español por parte de los estudiantes extranjeros. Asimismo, adquiere cada vez mayor importancia, debido a su posible aplicabilidad en la enseñanza o en la creación de material de clase.

El aprendizaje de léxico $y$, en consecuencia, la disponibilidad léxica para un estudiante de E/LE, se refiere a "un proceso complejo, que tiene sus propias características y que es fundamental en el aprendizaje de una lengua” (Morante, 2005, p. 7). Así también, se ha señalado que los estudiantes de E/LE se quejan de no poder expresar lo que quieren decir, porque no cuentan con el léxico al momento de tener que expresar algo, lo que produce problemas en la comprensión y en la comunicación y, por ende, en su aprendizaje (Higueras, citado en Morante, 2005).

En otro orden de cosas, desde la sociolingüística, se ha destacado no solo que las mujeres serían más sensibles que los hombres a la norma prestigiosa, sino, además, que ellas

\footnotetext{
$\S$ De aquí en adelante, se utilizará el término segunda lengua (L2). La diferencia radica en que el estudio de una lengua extranjera se produce en un contexto formal de enseñanza, no necesariamente en el país en que se habla la lengua meta, esto es, en un entorno heteroglósico. La adquisición de una segunda lengua implica un proceso de inmersión en un entorno homoglósico (Moreno Fernández, 2010). Esta investigación plantea un contexto mixto (de aprendizaje en inmersión), sin embargo, para evitar confusiones, se hará referencia siempre a la enseñanza de una L2.
} 
tendrían habilidades verbales mayores y mejores que estos. Dichas habilidades se verían reflejadas, por ejemplo, en el uso de un repertorio de variantes más amplio o el manejo de unos recursos estilísticos. De manera concreta, Moreno Fernández (2009, p. 45) postula que "las mujeres disponen de una capacidad neurofisiológica verbal que se puede manifestar en formas de diferencias sociolingüísticas, como el uso de un repertorio de variantes más amplio o el manejo de unos recursos estilísticos más ricos que los hombres”.

De lo anterior se derivan las preguntas que motivan esta investigación, a saber, ¿cuál es la disponibilidad léxica escrita de los hablantes extranjeros que estudian español como segunda lengua, al inicio de su inmersión? Al identificar el léxico disponible, ¿existe diferencia de este léxico disponible entre hombres y mujeres? Finalmente, ¿se puede determinar un léxico disponible común que comprenda a ambos géneros para facilitar el aprendizaje de la lengua? Desde los mencionados cuestionamientos, este estudio pretende determinar, de manera preliminar, si existen diferencias de sexo en la disponibilidad léxica escrita de los hablantes extranjeros que estudian español como segunda lengua en etapa inicial de inmersión. Para lograr este objetivo, se identificará el léxico disponible escrito en esta población de hablantes, se cuantificará dicho léxico y se asociará su disponibilidad con la variable externa sexo. Además, se determinará el léxico disponible común a la variable externa sexo, con el fin de facilitar y guiar la adquisición por nivel en los cursos de E/LE. Para esto, se creará una tabla que contenga el léxico común a todos los estudiantes, organizados por su nivel de español y por el léxico de las Nociones Específicas que serán utilizadas en este estudio, y su respectivo inventario léxico (Plan Curricular del Instituto Cervantes, 2007).

\section{Marco teórico}

\subsection{Variación sociolingüística del español: el léxico}

La variación a nivel léxico no ha recibido mucha atención por parte de los investigadores. López Morales (2004, p. 93) señala al respecto: "se comprende este cuasi silencio, si se tiene en cuenta que el nivel léxico -como el sintáctico- presenta unos problemas teóricos que es preciso encarar y solucionar antes de dar comienzo al trabajo 
propiamente sociolingüístico”. El autor hace referencia a la necesidad de demostrar que dos o más palabras constituyan parte del mismo conjunto de equivalencias, lo que significa que solo cuando dos o más palabras sean equivalentes lógicos o posean el mismo valor de verdad serán consideradas como paralelos semánticos. Moreno de Alba (2006) resume el mencionado problema como sigue:

Cuando se dice que la lengua española tiende a la unidad, mejor que a la diversidad, en lo que atañe a la fonología, es un tipo de afirmación que puede demostrarse con relativa facilidad: aunque fuera difícil establecer el número de sistemas fonológicos del diasistema de la lengua, debido precisamente a que se trata de sistemas y, por tanto, de inventarios cerrados, de elementos contables, llegará un momento en que sabremos cuántos son — no serán desde luego muchos - y cuál es el inventario y distribución de los fonemas en cada uno de ellos, que tampoco será notablemente diferente entre unos y otros. Este tipo de certezas no son posibles en el nivel del léxico. El carácter verdaderamente abierto del vocabulario lo impide (Moreno de Alba, 2006, p. 187).

El debate sobre la imposibilidad teórica de la sinonimia es algo que también destaca Moreno Fernández (2009, p. 32), pero argumenta que la sociolingüística variacionista se ha convertido casi en su defensora acérrima, al menos a nivel discursivo, aunque a la hora de demostrar si existe o no una neutralización de dos o más términos, la tarea se complica, sobre todo, cuando el uso está acompañado de valoraciones o connotaciones particulares o, bien, cuando el hablante tiene una intención comunicativa vinculada, por ejemplo, con el estilo lingüístico.

La variación lingüística, comprendida como inherente a las lenguas, se puede entender, según Moreno Fernández, como "la alternancia y la multivocidad” de los elementos léxicos-gramaticales según su significado semántico y relacionados con el contexto de uso pragmático, que buscan una "misma intención comunicativa u ocupan unos mismos espacios lingüísticos” (2010, p. 24). A partir de esto, surgen los conceptos de multivocidad y de alternancia. El primero está relacionado con más de un significado y con la diversidad de contextos en que pueden ser usados; el segundo, se explica a partir de dos o más elementos que tienen el mismo significado y ocupan un mismo espacio lingüístico (ibid.).

La variación léxica en sociolingüística, se estudia, en la mayoría de los casos, a partir de las variantes de una misma variable, esto es, de los sinónimos que puede tener una misma palabra y que se usa como variación en contexto. En este sentido, Moreno Fernández explica 
que para "abordar el estudio de la variación léxica hay que demostrar la equivalencia de una serie de variantes léxicas" (2009, p. 33) y además hay que encontrar estas variantes en el uso realizado por los hablantes. La variación léxica en multivocidad conforma parte de una misma intención comunicativa, pero como variantes de una variedad léxica (Moreno Fernández, 2010). Este tipo de variación permite al hablante de una lengua seleccionar el término que le parezca más adecuado desde una perspectiva semántica y de uso contextual. Esta posibilidad de selección es la que contiene una carga semántico-gramatical, es decir, es la que tiene un matiz de significado distinto dentro del mismo contexto y campo semántico y, además, posibilita el desarrollo del inventario léxico que será tratado más adelante en este estudio, en razón de las "Nociones Específicas” (PCIC, 2007) y del léxico disponible."*

Calsamiglia y Tusón (1999) y Serrano (2011) postulan que el léxico constituye un marcador de pertenencia a un grupo, lo que tiene como consecuencia que se pueda estudiar el léxico de diferentes grupos dentro de una misma sociedad. En lo que respecta al presente estudio, es el factor sexo el que se supone resulta diferenciador grupal. ${ }^{\dagger \dagger}$ Así, es pertinente señalar que varios estudios han puesto de relieve que la mujer tiene habilidades verbales mayores y mejores que los hombres y que estas van más allá de las diferencias socioculturales. En este orden y según ya se señaló, las mujeres y hombres manifestarían diferencias en su repertorio lingüístico, lo que podría manifestarse en forma de diferencias sociolingüísticas, como el uso de un repertorio de variantes más amplio o el manejo de unos recursos estilísticos más ricos en comparación a los hombres, siempre y cuando se comparen entre los mismos grupos sociales (cf. Moreno Fernández, 2009). Estos hallazgos son los que nos hacen suponer que existirían diferencias de sexo en el proceso de adquisición de una segunda lengua.

\subsection{Algunas precisiones sobre la enseñanza de español como segunda lengua}

\footnotetext{
${ }^{\star *}$ Es importante en este punto aclarar la diferencia entre léxico disponible y léxico frecuente. El primero, se mide en relación con los centros de interés o nociones semántico-gramaticales de uso, así como a los campos asociativos y las unidades temáticas que unen a un grupo léxico y lo diferencia de otro con respecto del contexto en que pueden ser usadas, corresponde a palabras que siempre están activas para ser utilizadas y que son menos frecuentes, pero no menos usuales; en cambio, el léxico frecuente se determina por el factor frecuencia y delimita a palabras con un alto uso gramatical y un bajo contenido semántico, que sirven indistintamente de un tema concreto u otro (Gallego, 2014).

${ }^{\Uparrow}$ Estudios prototípicos en el ámbito hispánico que se han encargado de la variación léxica son los de Ávila (1997) y Escoriza (2002 y 2004) para la comunidad de habla de Cádiz.
} 
Para el presente estudio "lengua estándar" será entendida como "lengua general”, es decir, como el:

Conjunto de elementos que son comunes a todas las modalidades de español y al que se le puede aplicar tanto la etiqueta de 'español general' como la de 'español estándar'. Aquí se incluirían el mínimo de 17 fonemas consonánticos compartidos, el sistema vocálico plenamente común, el léxico estructurado y fundamental para el español de cualquier latitud y unas bases gramaticales compartidas (Moreno Fernández, 2010, p. 9).

Los modelos de enseñanza de español como segunda lengua han podido unificarse en mayor o menor medida en relación con el "español estándar", ya que debido a la gran cantidad de variedades de la lengua, se hace necesario un modelo común para poder llevar a cabo la tarea de la adquisición del español (Moreno Fernández, 2010).

El Instituto Cervantes está inmerso en las políticas lingüísticas de la enseñanza del español como segunda lengua a través de su Plan Curricular, en cuyo marco desarrolla inventarios de Nociones Generales y de Nociones Específicas. En esta investigación, solo se trabajará con las segundas, que "son las que tienen que ver con detalles más concretos del <aquí y ahora> de la comunicación” (PCIC, 2007); ${ }^{\dagger \dagger}$ específicamente, con la Noción 2 Individuo: dimensión perceptiva y anímica, en la subdivisión “Carácter y personalidad” y la Noción Específica 4: Relaciones personales, en la subdivisión "Relaciones familiares". Las nociones seleccionadas se relacionan con el tipo de corpus analizado: en concreto, los estudiantes extranjeros de ELE en la sección de Redacción de la prueba de diagnóstico, debían crear un ensayo desde su perspectiva personal y conforme a un cuento o extracto de cuento preestablecido, en el que tenían como propósito desarrollar el tema de la vida familiar en Latinoamérica (cf. 3.1). La instrucción permite suponer que son las nociones antes mencionadas las que resultarán provechosas para el análisis.

\subsubsection{El estudio del léxico}

\footnotetext{
\$ Las Nociones Específicas están organizadas en un inventario léxico de la siguiente manera (PCIC, 2007, Nociones Específicas): 1) Individuo: dimensión física, 2) Individuo: dimensión perceptiva y anímica, 3) Identidad personal, 4) Relaciones personales, 5) Alimentación, 6) Educación, 7) Trabajo, 8) Ocio, 9) Información y medios de comunicación, 10) Vivienda, 11) Servicios, 12) Compras, tiendas y establecimientos, 13) Salud e higiene, 14) Viajes, alojamiento y transporte, 15) Economía e industria, 16) Ciencia y tecnología, 17) Gobierno, política y sociedad, 18) Actividades artísticas, 19) Religión y filosofía, 20) Geografía y naturaleza.
} 
Antes de la década del setenta, el léxico tenía un rol secundario respecto de la gramática, ya que no se concebía como un aspecto básico en la enseñanza de segundas lenguas. Así lo plantea Morante (2005) cuando señala que lo relevante para el aprendiz [era] aprender la pronunciación y la sintaxis. Recién desde 1970, el léxico se convirtió en una categoría en sí misma y se postularon oportunidades para desarrollar estrategias que permitieran interpretar y utilizar la lengua "como lo hacen los nativos" (Morante, 2005, p. 22). En consecuencia, se introduce el vocabulario en el aprendizaje en relación con el uso por parte de los aprendientes.

Luego, desde 1980, se trabaja con un enfoque en la gestión del aprendizaje, en cuyo caso la adquisición del vocabulario por parte del aprendiente de EL2, se considera como un proceso de carácter cualitativo antes que cuantitativo y, por lo tanto, se aborda el lexicón en sus aspectos dinámicos, con la intención de encontrar reglas y regularidades (Morante, 2005).

Los datos anteriores permiten inferir que, actualmente, en el estudio del léxico, es esencial comprender la disponibilidad léxica, puesto que no es fija, sino que podría variar según algunos factores externos, como el contexto de adquisición o aprendizaje, el sexo de los aprendientes, entre otros aspectos.

\section{Metodología}

\subsection{Corpus}

El corpus corresponde a treinta y cuatro pruebas de diagnóstico realizadas por el Programa de Español como Lengua Extranjera de la Pontificia Universidad Católica de Santiago de Chile a los alumnos extranjeros de intercambio llegados al país en agosto 2015, cuyo propósito era estudiar español como segunda lengua en los cursos impartidos por el Departamento de Español de la Facultad de Letras de dicha universidad, durante el segundo semestre de ese año. Esta prueba de diagnóstico consta de cinco secciones de evaluación, a saber: 1) formas verbales en combinación y uso de preposiciones, 2) completación de diálogo, 3) vocabulario, comprensión lectora, parafraseo, 4) uso de conectores y 5) redacción. 
En esta investigación solo se trabajará con la sección de redacción, ${ }^{\S \S}$ debido a que se trata de un trabajo preliminar que, en términos generales, pretende indagar en la existencia de diferencias de sexo en la disponibilidad léxica escrita de los hablantes extranjeros que estudian español como segunda lengua en etapa inicial de inmersión. Es en el contexto escrito -no en el oral- donde los hablantes utilizarían un vocabulario más abundante, formal y adecuado al contexto académico.

Cabe señalar que la prueba de diagnóstico realizada a los alumnos de intercambio es un instrumento validado por el Instituto Cervantes y funciona de acuerdo con las pautas de enseñanza del Marco Común Europeo (Consejo de Europa, 2002).

\subsection{Población y muestra}

La población corresponde a los 51 alumnos de intercambio llegados a Chile en agosto 2015 para cursar el segundo semestre académico y cuyo propósito es estudiar español como segunda lengua en contexto de inmersión. La muestra se delimitará a 17 mujeres y 17 hombres pertenecientes a este grupo de hablantes extranjeros, cuyo nivel de español oscila entre niveles intermedio y avanzado. Se trata de informantes que tienen entre 18 y 30 años, todos estudiantes universitarios.

Para la selección de informantes, se utilizó la muestra por cuotas con afijación uniforme (López Morales, 1994, p. 58-59). Así, fueron seleccionados cuatro alumnos de nivel intermedio de español y 13 alumnos de nivel avanzado de español por cada sexo, a saber, ocho alumnos de nivel intermedio de español y veintiséis estudiantes de nivel avanzado, en total, treinta y cuatro pruebas de diagnóstico. El número de alumnos por nivel de español es un dato que solo será utilizado tangencialmente, ya que no es el foco de este estudio. Asimismo, se tendrá en consideración la lengua materna y el nivel de español de los sujetos como dato referencial para explicar posibles hallazgos de esta investigación. Dentro de los

\footnotetext{
${ }_{\S}^{\S}$ Para esta prueba en específico, la sección de redacción contuvo dos fragmentos de textos literarios, "Tragedia” de Vicente Huidobro y "Crianzas" de Cristina Peri Rossi, de los que el estudiante debió elegir uno y escribir un comentario de mínimo veinte líneas, en el que se debe expresar las implicaciones del cuento con relación a la vida familiar latinoamericana. El fragmento de Huidobro, en adelante "Texto 1", consta de 14 líneas y el fragmento de Peri Rossi, en adelante "Texto 2", consta de 10 líneas. Ambos fueron tomados de la compilación de Juan Epple y James Heinrich "Para empezar, cien microcuentos hispanoamericanos" (1990). La elección de textos literarios no incide en la producción escrita de los informantes, dado que se trata de textos de fácil lectura, elegidos por sus temáticas antes que por el género al que pertenecen. Se trata, además, de un instrumento validado por el Instituto Cervantes y que funciona según las pautas de enseñanza del Marco Común Europeo (Consejo de Europa, 2002).
} 
informantes, además, existen diversos países de origen, los que fueron agrupados según lengua materna, como muestra la Tabla 1.

Tabla 1. Distribución de informantes pertenecientes a la muestra, según sexo, lengua materna y nivel de español al inicio de su inmersión

\begin{tabular}{|c|c|c|c|c|c|}
\hline \multirow[t]{2}{*}{ Variables } & \multirow[t]{2}{*}{ Sexo } & \multicolumn{2}{|c|}{ Nivel de español } & \multicolumn{2}{|c|}{ Lengua materna } \\
\hline & & $\mathrm{H}$ & $\mathrm{M}$ & $\mathrm{H}$ & $\mathrm{M}$ \\
\hline Hombre & 17 & & & & \\
\hline Mujer & 17 & & & & \\
\hline Intermedio & & 4 & 4 & & \\
\hline Avanzado & & 13 & 13 & & \\
\hline Inglés & & & & 2 & 5 \\
\hline Francés & & & & 3 & \\
\hline Portugués & & & & 1 & 1 \\
\hline Alemán & & & & 1 & 5 \\
\hline Sueco & & & & 2 & 1 \\
\hline Noruego & & & & & 2 \\
\hline Suizo & & & & 2 & \\
\hline Danés & & & & 2 & 2 \\
\hline Holandés & & & & 1 & \\
\hline Chino & & & & 2 & 1 \\
\hline Inglés- Maorí & & & & 1 & \\
\hline Totales & 34 & 17 & 17 & 17 & 17 \\
\hline
\end{tabular}

\subsection{Procesamiento de los datos}

Los datos relevantes para la presente investigación son aquellos correspondientes al léxico disponible escrito de la sección "Redacción” de la prueba de diagnóstico tomada a los informantes. El análisis del léxico disponible se correlacionará con la variable externa sexo. Los informantes escribieron sobre la vida familiar en América Latina, para lo que, además, se tendrán en cuenta los datos de eventuales variables no controladas como el nivel de español (intermedio o avanzado) y la lengua materna (once variedades de lengua).

El léxico disponible será delimitado a través de las Nociones Específicas señaladas en el Plan Curricular del Instituto Cervantes (PCIC, 2007). Se utilizará el léxico de dos nociones Específicas: [02] Individuo, dimensión perceptiva y anímica: Carácter y 
personalidad y [04] Relaciones personales: Relaciones familiares. Además, se agregará al léxico disponible antes dicho, aquel presente en el corpus que no se encuentre inventariado en las Nociones Específicas ya especificadas.

Con el léxico seleccionado, se hará una matriz en la que, en primer lugar, se pueda realizar un análisis descriptivo, por informante y su respectivo nivel de español y lengua materna correlacionado con la variable externa sexo. Una vez identificado el léxico disponible, se confeccionará un análisis comparativo para conocer las diferencias de léxico entre hombres y mujeres; y, finalmente, determinar si existe una disponibilidad léxica común a ambos sexos, por nivel de español.

\section{Presentación, análisis y discusión de resultados}

A partir del concepto de léxico disponible, se presenta un análisis general descriptivo de dicho léxico, por una parte, supeditado a las Nociones Específicas 2 y 4 (en adelante, NE2 y NE4) y al inventario léxico según nivel de referencia de español (A1, A2, B1, B2) entregado por el Plan Curricular del Instituto Cervantes (2007). Por otra parte, relacionado con el léxico no inventariado en estas nociones semántico-gramaticales, pero que será incluido en el análisis como aporte de investigación; y, por último, sujeto a las posibles variables extrañas que caracterizan a los treinta y cuatro informantes, a saber, el nivel de diagnóstico de español como segunda lengua en su proceso inicial de inmersión y su lengua materna. Este análisis descriptivo tiene por objetivo presentar resultados generales, para identificar y caracterizar el léxico disponible escrito en esta población de hablantes, según nivel de español y sexo. ${ }^{* * *}$

Para comenzar, se expondrá el listado del inventario léxico por Noción Específica 2 Individuo: dimensión perceptiva y anímica: Carácter y personalidad (NE2), Noción Específica 4: Relaciones personales: Relaciones familiares (NE4) y nivel de referencia de español (A1, A2, B1, B2). Asimismo, se dará a conocer el léxico no inventariado (LNI) en las Nociones y niveles de referencias seleccionados, y que fue incluido en el presente análisis, por orden de aparición. Dentro del inventario léxico, se tomó en cuenta solo el perteneciente

\footnotetext{
${ }^{* * *}$ Hallazgos más específicos serán presentados en Galdames, Toledo y Guerrero (m/i).
} 
a las categorías gramaticales de sustantivos y adjetivos,,$^{\dagger \dagger}$ a fin de poder realizar la caracterización semántico-gramatical del léxico utilizado en el corpus, en función del contexto específico de uso de cada Noción y nivel.

Tabla 2. Distribución de léxico por NE 2, NE4 y nivel de referencia de español

\begin{tabular}{|c|c|c|c|c|c|}
\hline NE2 & & & NE4 & & \\
\hline A1 & $\mathrm{A} 2$ & LNI & A1 & $\mathrm{A} 2$ & LNI \\
\hline Simpático & Carácter & Feliz & Familia & Papá & $\begin{array}{l}\text { Esposo } \\
(\mathrm{a})^{+4}\end{array}$ \\
\hline Antipático & Optimista & Malo & Padres $^{\S \S \S}$ & Mamá & \\
\hline Inteligente & Abierto & Bueno & Padre & Hijo único & \\
\hline Trabajador & Reservado & & Madre & $\begin{array}{l}\text { Hermano } \\
\text { gemelo }\end{array}$ & \\
\hline Alegre & Tranquilo & & Hijo (a) & Ex marido & \\
\hline Serio & Nervioso & & Hermano & Ex mujer & \\
\hline Tímido & Generoso & & Tío & & \\
\hline Sociable & Egoísta & & Sobrino & & \\
\hline & Amable & & Abuelo & & \\
\hline & Agradable & & Nieto & & \\
\hline & Tolerante & & Primo & & \\
\hline & & & Marido & & \\
\hline & & & Mujer & & \\
\hline & & & Pareja & & \\
\hline & & & Novio & & \\
\hline B1 & $\mathrm{B} 2$ & LNI & B1 & B2 & LNI \\
\hline Optimismo & Ambición & (In)fiel & $\begin{array}{l}\text { Matrimonio } \\
* * * *\end{array}$ & Cónyuge & $\begin{array}{l}\text { Relaciones } \\
\text { familiares }\end{array}$ \\
\hline Pesimismo & Arrogancia & $\begin{array}{l}\text { Personalidad } \\
\text { tit }\end{array}$ & $\begin{array}{l}\text { Matrimonio } \\
\text { homosexual }\end{array}$ & Cuñado & $\begin{array}{l}\text { Relación } \\
\text { especial }\end{array}$ \\
\hline
\end{tabular}

†i† Esta decisión se sustenta, entre otros, en los hallazgos de Sánchez (2005), quien estudia la naturaleza gramatical del léxico disponible en hablantes de español como lengua extranjera y concluye que la categoría predominante en la disponibilidad léxica es la sustantiva.

H El ítem léxico ‘esposo(a)' corresponde al nivel C1.

$\S \S$ El ítem léxico 'padres' se refiere de manera genérica a padre y madre.

${ }^{* * * *}$ El ítem léxico 'matrimonio' es utilizado por informante que ha sido calificado para entrar a nivel intermedio en inmersión.

${ }^{\dagger \dagger \dagger}$ El ítem léxico 'personalidad' corresponde al nivel C2. 


\begin{tabular}{|c|c|c|c|c|c|}
\hline (In)tranquilidad & (In)constancia & Divertido & $\begin{array}{l}\text { Matrimonio } \\
\text { heterosexual }\end{array}$ & Suegro & $\begin{array}{l}\text { Situación } \\
\text { familiar }\end{array}$ \\
\hline (Im)paciencia & Cobardía & Triste & $\begin{array}{l}\text { Pareja de } \\
\text { hecho }\end{array}$ & Yerno & $\begin{array}{l}\text { Vida } \\
\text { familiar }\end{array}$ \\
\hline Sincero & Curiosidad & Extrovertido & Madre soltera & Nuera & $\begin{array}{l}\text { Buenas } \\
\text { relaciones }\end{array}$ \\
\hline (Im) paciente & Egoísmo & Cerrado & $\begin{array}{l}\text { Niño } \\
\text { adoptado }\end{array}$ & Bisabuelo & Hijito(a) \\
\hline Introvertido & Generosidad & Tradicional & & Tatarabuelo & Amante \\
\hline Vago & (Im)puntualidad & Encantador & & Mellizos & \\
\hline Travieso & (Ir)responsabilidad & Moderno & & Trillizos & \\
\hline (In)seguro & (In)sensibilidad & Libre & & $\begin{array}{l}\text { Madre } \\
\text { de alquiler }\end{array}$ & \\
\hline Conservador & Seriedad & Fidelidad & & Niño probeta & \\
\hline \multirow[t]{16}{*}{ Arrogante } & (In) sinceridad & Envidioso & & $\begin{array}{l}\text { Familiar } \\
\text { cercano }\end{array}$ & \\
\hline & Ternura & Cruel & & $\begin{array}{l}\text { Familiar } \\
\text { lejano }\end{array}$ & \\
\hline & Timidez & & & $\begin{array}{l}\text { Familia } \\
\text { numerosa }\end{array}$ & \\
\hline & Valentía & & & $\begin{array}{l}\text { Familia } \\
\text { adoptiva }\end{array}$ & \\
\hline & Ambicioso & & & $\begin{array}{l}\text { Padre } \\
\text { adoptivo }\end{array}$ & \\
\hline & Callado & & & Hijo adoptivo & \\
\hline & Cobarde & & & Maternidad & \\
\hline & Constante & & & Paternidad & \\
\hline & Curioso & & & & \\
\hline & Discreto & & & & \\
\hline & Tacaño & & & & \\
\hline & Tierno & & & & \\
\hline & (Ir)responsable & & & & \\
\hline & Susceptible & & & & \\
\hline & Apasionado & & & & \\
\hline & Solidario & & & & \\
\hline
\end{tabular}


A partir del inventario anterior, por nivel de referencia y Noción, se buscó cada ítem léxico en el corpus y se agregó, por orden de aparición, aquel pertinente en cuanto a campo semántico y categoría, el que se presenta a continuación de manera descriptiva y, luego, a partir del análisis de frecuencias, con estadística descriptiva. ${ }^{+1+}$

De NE2, A1, hubo aparición en uso de los siguientes ítems léxicos: ${ }^{\S \S \S}$ Inteligente (3/1), Alegre (4/2), Serio (2/1), Tímido (1/1). De NE2, A2, hubo aparición en uso de los siguientes ítems léxicos: Amable (1/1), Agradable (1/1). Los ítems léxicos no inventariados fueron los siguientes: Feliz (9/6), Malo (1/1), Bueno (8/4). De NE2, B1, hubo aparición en uso de la palabra Conservador (3/3). De la NE2, B2, no hubo aparición de ninguno de los ítems léxicos inventariados. Los ítems léxicos no inventariados fueron los siguientes: (In)fiel (11/7), Personalidad (2/1), Divertido (2/2), Triste (1/1), Extrovertido (1/1), Cerrado (1/1), Tradicional (3/3), Encantador (2/2), Moderno (1/1), Libre (1/1), Fidelidad (1/1), Envidioso $(1 / 1)$, Cruel (1/1).

De NE4, A1, hubo aparición en uso de los siguientes ítems léxicos: Familia (24/13), Padres (25/9), Padre (2/2), Madre (54/17), Hijo(a) (54/12), Abuelo(a) (3/1), Marido (23/10), Mujer $^{* * * * *}$ (10/8). De NE4, A2, hubo aparición en uso del léxico Mamá (3/1). El léxico no inventariado fue Esposo(a) (4/4). De NE4, B1, hubo aparición en uso del ítem léxico Matrimonio (3/2). De NE4, B2, no hubo aparición de ninguno de los ítems léxicos inventariados. Por su parte, los ítems léxicos no inventariados fueron los siguientes: Relaciones familiares (4/2), Relación especial (1/1), Situación familiar (1/1), Vida familiar (14/10), Buenas relaciones (1/1), Hijito(a)(s) (1/1), Amante (8/5).

Es importante señalar que en el corpus referente a la NE2 se seleccionó el léxico correspondiente a la caracterización de personalidad de individuos, familias, sociedades y culturas, dejando fuera de esta tabla y de la cuantificación general cualquier palabra correspondiente a otra noción.

\footnotetext{
H\$ Es preciso señalar que se realizaron pruebas estadísticas, pero ningún análisis resultó estadísticamente significativo. ${ }^{\S \S \S}$ Se pondrá entre paréntesis la cantidad de repetición del léxico más la cantidad de informantes que la utilizaron (X/Y). ***** El léxico 'mujer' se tomó en cuenta para el siguiente análisis cuando era contrapuesto a 'marido' y se dejó fuera cuando era contrapuesto a 'hombre'.
} 


\subsection{Datos cuantitativos de léxico disponible correlacionado con variable externa $\operatorname{sexo}$}

El corpus de treinta y cuatro redacciones corresponde a trece mujeres y trece hombres en inicio de nivel avanzado y cuatro mujeres y cuatro hombres en inicio de nivel intermedio. Se realizó un análisis comparativo, con el fin de relacionar el léxico disponible y la variable sexo dentro de cada nivel, no entre niveles, ya que la cantidad de informantes es distinta. Se comienza con la descripción de los datos de los informantes de nivel intermedio (NI).

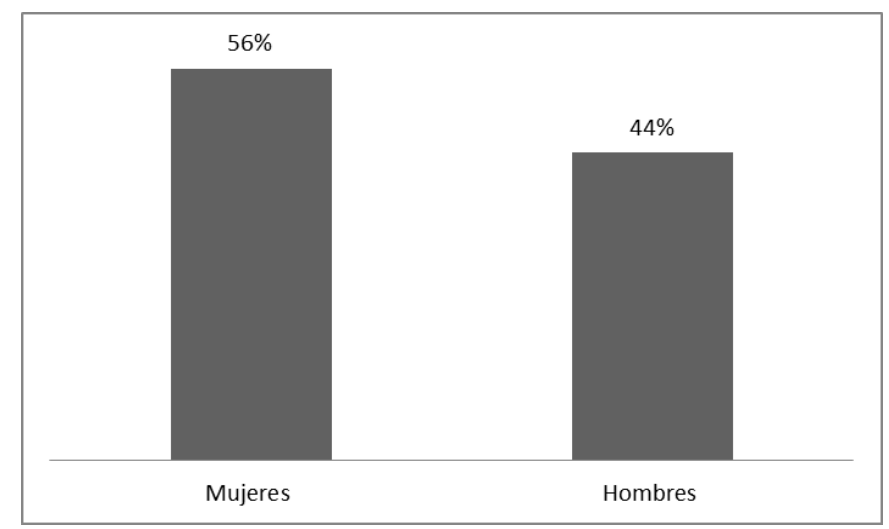

Gráfico 1. Léxico total por sexo, informantes NI, léxico nivel básico

El gráfico precedente muestra los porcentajes totales de léxico utilizado por los informantes de nivel intermedio, en relación con la variable sexo. Como se observa, los hombres utilizan un $44 \%$ del léxico disponible, mientras las mujeres usan el $56 \%$ de este. A pesar de haber diferencia entre ambos sexos, no comprobadas estadísticamente, al menos desde el punto de vista descriptivo las mujeres tienen un mayor output léxico. La diferencia, como puede verse, no es sustancial, más aún al tratarse solo de ocho informantes, cuatro hombres y cuatro mujeres, quienes en total tienen una aparición en uso de 43 ítems léxicos, 24 las mujeres y 19 los hombres.

Las mujeres, en general, se caracterizan por utilizar léxico perteneciente a NE4. Esto ocurre excepto para las informantes cuya lengua materna es el alemán, que nombran el adjetivo 'Feliz'. Por su parte, los informantes hombres tampoco utilizan adjetivos para expresarse semánticamente, salvo un informante cuya lengua materna es el sueco, quien en su redacción utiliza cuatro ítems léxicos de NE2, correspondientes a los adjetivos 
'Inteligente' tres veces y al adjetivo 'Agradable' una vez. El mismo sujeto no usa ningún ítem léxico de NE4. Este informante es el único de los treinta y cuatro que utiliza solo léxico de NE2.

A continuación, se darán a conocer los gráficos con los datos de los informantes de nivel avanzado (NA).

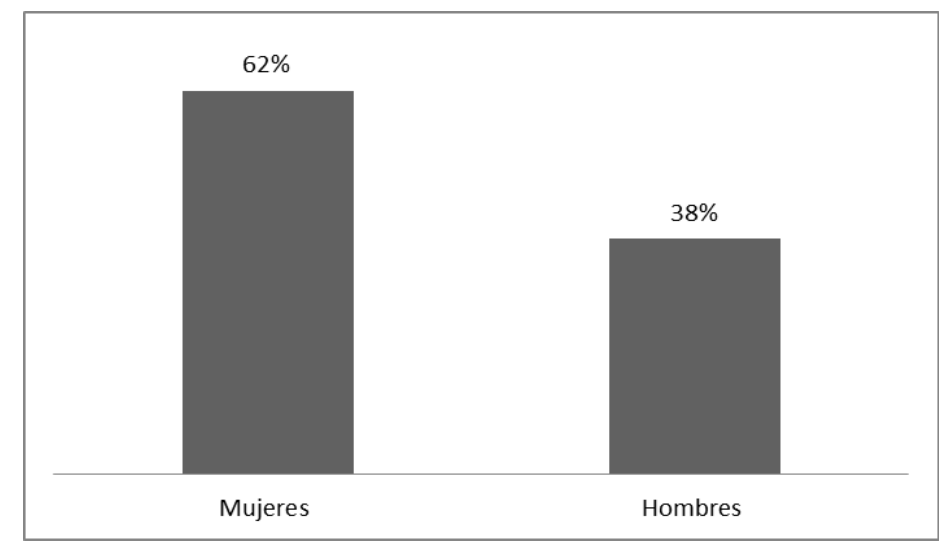

Gráfico 2. Léxico total por sexo, informantes NA, léxico nivel básico

El gráfico precedente muestra el total en porcentajes del léxico de nivel básico, comparado por sexo en informantes de nivel avanzado. En este caso, nuevamente, las mujeres registran mayor disponibilidad léxica que los hombres. En concreto, los textos redactados por informantes mujeres obtienen un $62 \%$ y los hombres un $38 \%$ de utilización de léxico disponible. En este gráfico, queda incluido el léxico no inventariado registrado en el corpus.

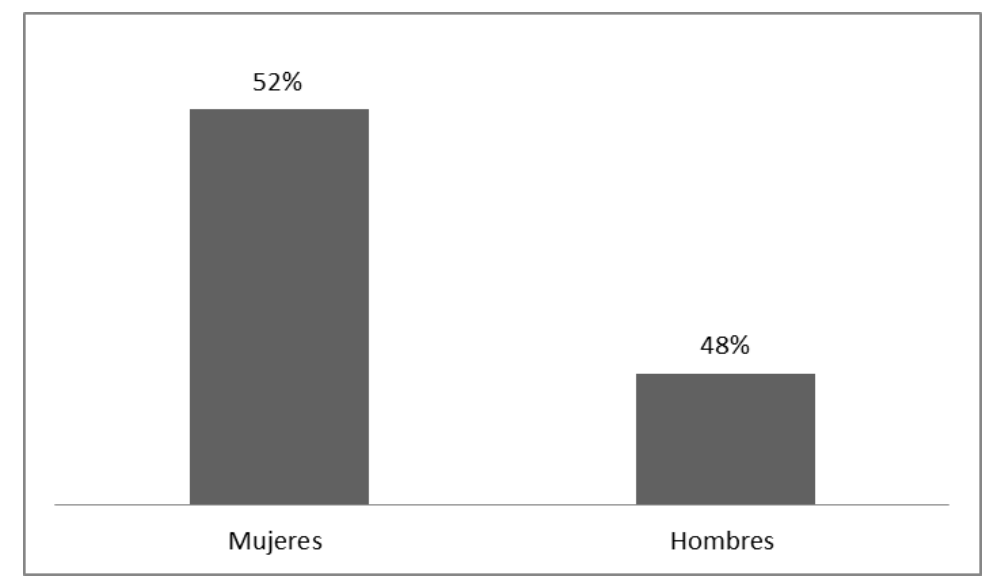

Gráfico 3. Léxico total por sexo, informantes NA, léxico nivel intermedio 
El gráfico 3, como puede apreciarse, muestra el total en porcentajes del léxico nivel intermedio comparado entre hombre y mujer de informantes de nivel avanzado, en el que se puede ver un $52 \%$ que representa a las mujeres y un $48 \%$ a los hombres. En este nivel, nuevamente, las mujeres registran mayor empleo del léxico disponible. Está incluido, además, el léxico no inventariado del corpus.

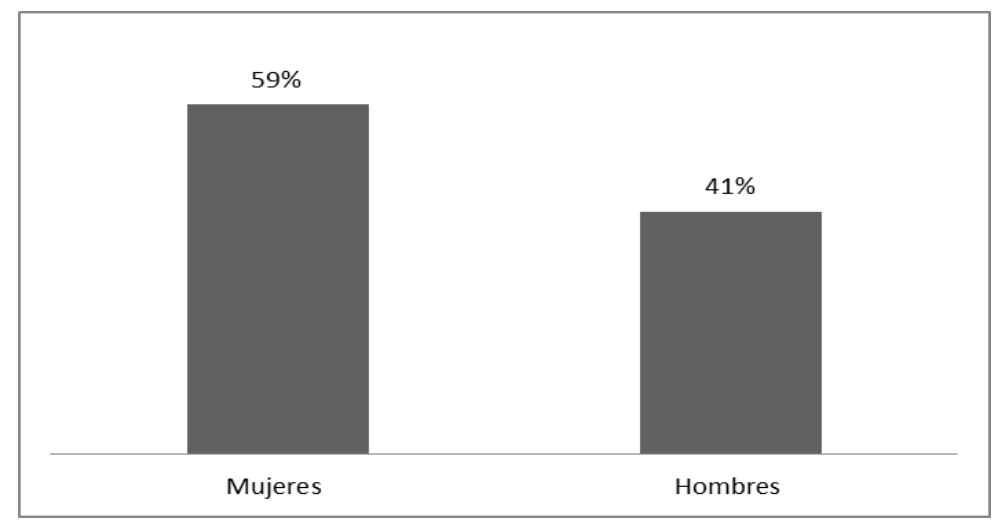

Gráfico 4. Léxico total por sexo, informantes NA, léxico nivel básico e intermedio

El gráfico número 4 muestra en porcentajes el total del léxico disponible utilizado, comparado entre hombres y mujeres en informantes de nivel avanzado. Está incluido el léxico nivel básico e intermedio, así como también el léxico no inventariado del corpus. Se puede apreciar que las mujeres tienen un $59 \%$ de los términos y los hombres un $41 \%$ de ítems léxicos en uso.

Los gráficos anteriores muestran la disponibilidad léxica de los veintiséis informantes de nivel avanzado, comparados por sexo, trece mujeres y trece hombres. Primero, se puede decir que las mujeres utilizan más el léxico de nivel básico que los hombres, con un 62 \% que corresponde a 117 palabras, por sobre un $38 \%$ que corresponde a 73 palabras, mientras en el léxico de nivel intermedio la diferencia es mucho menor, $52 \%$ las mujeres y los hombres $48 \%$. Es en el empleo del léxico básico donde hay más diferencia, sobre todo en NE2, donde los hombres no utilizan ningún ítem léxico de esta Noción, salvo dos apariciones de 'Feliz', correspondiente al léxico no inventariado; mientras las mujeres usan siete ítems léxicos, con un total de veintiún apariciones: 4 'Alegre', 2 'Serio'; 1 'Tímido'; 1 'Amable'; más los no inventariados, 5 'Feliz'; 1 'Malo' y 7 'Bueno'. Cabe señalar que la transferencia del idioma portugués no resulta relevante en relación con los porcentajes; sin embargo, es el 
hablante de dicha lengua el único que utiliza dos ítems léxicos de NE2 (2 'Alegre' y 2 'Serio'), lo que podría deberse a que la transferencia lingüística y la semejanza ente los idiomas le den mayor seguridad lingüística para atreverse a usar adjetivos. Respecto de NE4, como se vio en los gráficos anteriores, la aparición y repetición de léxico es más similar entre hombres y mujeres, ya que la diferencia sustancial se hace en NE2.

Como resultado preliminar respecto del léxico inventariado en el nivel básico, usado por los informantes que comienzan su inmersión en nivel avanzado, se podría señalar que, al haber mayor disponibilidad léxica, las mujeres podrían tener una mayor facilidad para asimilar la adquisición de adjetivos que los hombres, así como que los hombres tenderían a realizar más repeticiones de sustantivos. Esto ocurre, principalmente, en el léxico disponible que ya está asimilado, a saber, el del nivel básico. De esta manera, se puede comprobar que existe una diferencia en la adquisición del léxico disponible entre hombres y mujeres, al menos desde el análisis descriptivo de los datos. De cualquier forma, se trata de hipótesis explicativas tentativas para intentar justificar los hallazgos de esta investigación. Así, la realización de estudios de adquisición en el campo de la lingüística aplicada o en la psicolingüística podría validar o refutar nuestros resultados.

En el léxico de nivel intermedio existe menos porcentaje de diferencia entre hombres y mujeres que en el léxico de nivel básico: un $48 \%$ equivalente a 30 palabras, los primeros, y un $52 \%$ equivalente a 33 palabras las segundas, como ya se señaló. En la NE2 cinco hombres utilizan seis ítems léxicos con una repetición total de diez veces: 2 'Conservador'; 4 '(In)fiel; 1 'Encantador'; 1 'Fidelidad'; 1 'Envidioso'; 1 'Cruel' y seis mujeres utilizan 11 ítems léxicos con un total de veintiún apariciones: 1 'Conservador'; 7 '(In)fiel; 2 'Personalidad'; 2 'Divertido'; 1 'Triste'; 1 'Extrovertido'; 1 'Cerrado'; 3 'Tradicional'; 1 'Encantador'; 1 'Moderno'; 1 'Libre'. Lo anterior llama la atención respecto de la variedad de léxico disponible, ya que los hombres utilizan menos variedad léxica que las mujeres; sin embargo, la repetición de léxico disponible general entre ambos sexos es semejante, con algunos casos que se escapan de la media.

Mientras en la NE4 de nivel intermedio, los hombres utilizan 3 ítems léxicos con una repetición de veinte veces: 3 'Relaciones familiares'; 11 'Vida familiar'; 6 'Amante', las mujeres utilizan 8 ítems léxicos con una repetición de 12 veces: 1 'Relación familiar'; 1 'Relación especial'; 1 'Situación familiar'; 3 'Vida familiar'; 1 'Buenas relaciones'; 1 
'Hijito'; 2 'Amante'. En esta Noción, es importante hacer notar que la disponibilidad léxica entre uno y otro grupo varía bastante. Sobre todo llama la atención que los hombres utilicen solo 3 ítems léxicos en contraste con 12 de las mujeres, los que son repetidos en total 20 veces. Esto puede demostrar que el léxico disponible no es suficiente para lograr una buena comunicación, viéndose estos con pocas herramientas para poder expresar significado, lo que los obliga a repetir aquellas palabras con las que se sienten seguros.

El gráfico número 4 muestra los porcentajes totales comparados del léxico utilizado por los informantes de nivel avanzado, incluido el léxico básico y el léxico intermedio, donde se puede apreciar que las mujeres tienen un total del $59 \%$ y los hombres un $41 \%$ del uso de léxico disponible en las Nociones seleccionadas. Estos porcentajes totales son relevantes para confirmar descriptivamente la primera hipótesis: existe diferencia de léxico disponible entre hombres y mujeres al inicio de la inmersión del nivel avanzado, de adquisición de español como segunda lengua, así como también existe diferencia entre hombres y mujeres del nivel intermedio, pero en menor medida, esto es, un $57 \%$ las mujeres y un $43 \%$ los hombres. Entre otros autores, López Morales (2011) alude a que la "calidad de la escritura" está integrada por una serie de factores, por ejemplo, la riqueza léxica, la madurez sintáctica, los esquemas de cohesión y la coherencia discursiva. Asimismo, la disponibilidad léxica del hablante, apoya la amplitud y variedad del vocabulario la madurez sintáctica. En lo que se refiere al presente estudio, son las mujeres las que presentan mayor calidad de escritura.

A continuación, se presenta una tabla de las medias del léxico, nivel básico y nivel intermedio, utilizado por los informantes de nivel avanzado, comparado por sexo.

Tabla 3. Medias informantes NA comparada por sexo

\begin{tabular}{llll}
\hline Sexo Avanzado & & $\begin{array}{l}\text { Total léxico nivel } \\
\text { básico }\end{array}$ & $\begin{array}{l}\text { Total léxico nivel } \\
\text { intermedio }\end{array}$ \\
\hline Mujer & Media & 9,00 & 2,54 \\
\hline Hombre & Media & 5,62 & 2,31 \\
\hline Total & Media & 7,31 & 2,42 \\
\hline
\end{tabular}

Esta tabla da cuenta, principalmente, de dos cosas: primero, que tanto en hombres como en mujeres, hay un mayor uso del léxico correspondiente al nivel básico de adquisición de español como segunda lengua, las mujeres con una Media de 9,00 y los hombres con una 
Media de 5,62, frente al léxico del nivel intermedio, con mucho menor uso, donde las mujeres tienen una Media de 2,54 y los hombres una Media de 2,31, muy similar ente ellos. Estos resultados estadísticos revelan que la diferencia ocurre en la asimilación del léxico y en la seguridad lingüística de ambos grupos al momento de utilizar el léxico disponible. En teoría, ambos grupos conocen el léxico de igual manera, es el léxico del nivel básico el que debería estar más asimilado por ambos grupos de hablantes, mientras el léxico de nivel intermedio debería estar en proceso de asimilación y de práctica durante el nivel que le sigue, en este caso, el avanzado. Pero el grupo de informantes mujeres muestra un mayor uso, por lo tanto, una mayor asimilación del léxico disponible y una mayor seguridad lingüística para utilizarlo, en relación con los hombres, que muestran un menor uso y una tendencia a la repetición. ${ }^{\dagger \dagger \dagger i \dagger ~}$

En segundo lugar, se observa que la Media total, que incluye a ambos sexos es notablemente superior en el uso de léxico disponible del nivel básico, lo que reitera que el léxico más asimilado y que el informante le da mayor seguridad lingüística para usarlo, es el del nivel básico, con una Media de 7,31 frente a una Media de 2,42 en el léxico disponible contabilizado del nivel intermedio de adquisición de español como segunda lengua.

\subsection{Léxico disponible común a la variable externa sexo por nivel de español}

En este punto del análisis se detalla, a través de porcentajes, el uso de cada ítem léxico, distinguido por su pertenencia a un nivel de español y la Noción Específica en que está inventariado. Asimismo, se incluye el léxico no inventariado en las Nociones del Plan Curricular del Instituto Cervantes (2007) que fue encontrado en el corpus. El análisis está dividido en dos tablas, la primera para los informantes de nivel avanzado y la segunda para

\footnotetext{
$\prod^{\dagger \dagger \dagger \dagger}$ Estos resultados permiten pensar que entre las proyecciones del trabajo se halla en análisis comparativo de las formas lingüísticas más tradicionales que se consideran como "prestigiosas". De acuerdo con Silva-Corvalán (2001, p. 99), el uso de determinadas variables lingüísticas se considera prestigioso, es decir, adquiere un valor positivo, puesto que facilita el ascenso en la escala social y, además, se trata de formas lingüísticas aceptadas por las gramáticas normativas, por lo que, generalmente, son asociadas a la clase media alta culta. De acuerdo con Coates (2009), las investigaciones sociolingüísticas clásicas, como la de Labov (1966) en Nueva York y la de Trudgill (1974) en Norwich, que estudiaron variaciones lingüísticas en correlación con variables extralingüísticas, revelaron una clara estratificación social y dieron lugar a dos conceptos: el de prestigio y el de estigma. El primero se relaciona con las formas lingüísticas que suelen emplear los grupos sociales de más alto estatus, por lo que en el proceso de estandarización se llega a la noción de correcto. El estigma, por el contrario, se relaciona con las formas no estándares. Conforme creció el interés en el uso y permanencia de formas no estándares, estas variantes no estándares fueron denominadas vernáculas.
} 
los informantes de nivel intermedio. En cada tabla, se señala primero la Noción Específica 2 o 4, luego el nivel de español en que está inventariada. A continuación, se expone el léxico y la cantidad de veces que se repite la palabra, luego el porcentaje de aparición de este léxico disponible y en la última columna, el sexo en que esta tiene aparición: M si es utilizada solo por mujeres, $\mathrm{H}$ si es solo utilizada por hombres y M/H si es utilizada por ambos. Así, se busca llegar a determinar el léxico disponible común a la variable externa sexo, con el fin de facilitar y guiar la adquisición por nivel en los cursos de ELE. Es importante recordar que es parte del objetivo de esta investigación determinar un léxico común a ambos sexos, con la finalidad de que sirva como base para desarrollar herramientas de enseñanza y nivelación de la adquisición léxica.

Tabla 3. Porcentaje de aparición de léxico disponible común a la variable externa sexo en informantes NA

\begin{tabular}{|c|c|c|c|c|c|}
\hline $\begin{array}{l}\text { Noción } \\
\text { Específica }\end{array}$ & Nivel español & Ítem léxico & $\begin{array}{l}\text { N. } \\
\text { palabras }\end{array}$ & $\begin{array}{l}\text { Porcentaje } \\
\text { de aparición }\end{array}$ & Sexo \\
\hline 2 & A1 & Alegre & 4 & $1,6 \%$ & $\mathrm{M}$ \\
\hline 2 & A1 & Serio & 2 & $0,8 \%$ & $\mathrm{M}$ \\
\hline 2 & A1 & Tímido & 1 & $0,4 \%$ & $\mathrm{M}$ \\
\hline 2 & A2 & Amable & 1 & $0,4 \%$ & $\mathrm{M}$ \\
\hline 2 & No inventariada & Feliz & 7 & $2,8 \%$ & $\mathrm{M} / \mathrm{H}$ \\
\hline 2 & No inventariada & Malo & 1 & $0,4 \%$ & $\mathrm{M}$ \\
\hline 2 & No inventariada & Bueno & 7 & $2,8 \%$ & $\mathrm{M} / \mathrm{H}$ \\
\hline 4 & A1 & Familia & 18 & $7,1 \%$ & $\mathrm{M} / \mathrm{H}$ \\
\hline 4 & A1 & Padres & 24 & $9,5 \%$ & $\mathrm{M} / \mathrm{H}$ \\
\hline 4 & A1 & Padre & 1 & $0,4 \%$ & $\mathrm{H}$ \\
\hline 4 & A1 & Madre & 45 & $17,8 \%$ & $\mathrm{M} / \mathrm{H}$ \\
\hline 4 & A1 & Hijo(a) & 46 & $18,2 \%$ & $\mathrm{M} / \mathrm{H}$ \\
\hline 4 & A1 & Marido & 20 & $7,9 \%$ & $\mathrm{M} / \mathrm{H}$ \\
\hline 4 & A1 & Mujer & 10 & $3,9 \%$ & $\mathrm{M} / \mathrm{H}$ \\
\hline 4 & No inventariada (C1) & Esposo(a) & 3 & $1,2 \%$ & $\mathrm{M} / \mathrm{H}$ \\
\hline 4 & B2 & Matrimonio & 2 & $0,8 \%$ & $\mathrm{M}$ \\
\hline 4 & No inventariada & Relación familiar & 4 & $1,6 \%$ & $\mathrm{M} / \mathrm{H}$ \\
\hline 4 & No inventariada & Relación especial & 1 & $0,4 \%$ & $\mathrm{M}$ \\
\hline 4 & No inventariada & Situación familiar & 1 & $0,4 \%$ & $\mathrm{M}$ \\
\hline
\end{tabular}




\begin{tabular}{|c|c|c|c|c|c|}
\hline 4 & No inventariada & Vida familiar & 14 & $5,5 \%$ & $\mathrm{M} / \mathrm{H}$ \\
\hline 4 & No inventariada & Buenas relaciones & 1 & $0,4 \%$ & $\mathrm{M}$ \\
\hline 4 & No inventariada & Hijito(a) & 1 & $0,4 \%$ & $\mathrm{M}$ \\
\hline 4 & No inventariada & Amante & 8 & $3,2 \%$ & $\mathrm{M} / \mathrm{H}$ \\
\hline 2 & B1 & Conservador & 3 & $1,2 \%$ & $\mathrm{M} / \mathrm{H}$ \\
\hline 2 & No inventariada & (In)fiel & 11 & $4,3 \%$ & $\mathrm{M} / \mathrm{H}$ \\
\hline 2 & No inventariada (C2) & Personalidad & 2 & $0,8 \%$ & $\mathrm{M}$ \\
\hline 2 & No inventariada & Divertido & 2 & $0,8 \%$ & $\mathrm{M}$ \\
\hline 2 & No inventariada & Triste & 1 & $0,4 \%$ & $\mathrm{M}$ \\
\hline 2 & No inventariada & Extrovertido & 1 & $0,4 \%$ & $\mathrm{M}$ \\
\hline 2 & No inventariada & Cerrado & 1 & $0,4 \%$ & $\mathrm{M}$ \\
\hline 2 & No inventariada & Tradicional & 3 & $1,2 \%$ & $\mathrm{M}$ \\
\hline 2 & No inventariada & Encantador & 2 & $0,8 \%$ & $\mathrm{H}$ \\
\hline 2 & No inventariada & Moderno & 1 & $0,4 \%$ & $\mathrm{M}$ \\
\hline 2 & No inventariada & Libre & 1 & $0,4 \%$ & $\mathrm{M}$ \\
\hline 2 & No inventariada & Fidelidad & 1 & $0,4 \%$ & $\mathrm{H}$ \\
\hline 2 & No inventariada & Envidioso & 1 & $0,4 \%$ & $\mathrm{H}$ \\
\hline 2 & No inventariada & Cruel & 1 & $0,4 \%$ & $\mathrm{H}$ \\
\hline Total & & & 253 & $100 \%$ & $37 \%$ \\
\hline
\end{tabular}

Según el análisis reflejado en la tabla número 4, el léxico disponible para informantes de ambos sexos $(\mathrm{M} / \mathrm{H})$, de nivel avanzado, al inicio del período de inmersión, corresponde a 14 ítems léxicos entre 37 ítems léxicos aparecidos en uso en el corpus: 'Feliz' con una aparición del 2,8 \%, 'Bueno' con una aparición del 2,8 \%, 'Familia' con una aparición de 7,1 \%, 'Padres' con una aparición de 9,5 \%, 'Madre' con una aparición de 17,8 \%, 'Hijo' con una aparición de 18,2 \%, 'Marido' con una aparición de 7,9 \%, 'Mujer' con una aparición de 3,9 \%, 'Esposo' con una aparición de 1,2 \%, 'Relación familiar' con una aparición de 1,6 \%, 'Vida familiar' con una aparición de 5,5\%, 'Amante' con una aparición de 3,2\%, 'Conservador' con una aparición de 1,2 \% e '(In)fiel' con una aparición de 4,3 \%, los que en total corresponden al $37 \%$ del de léxico disponible utilizado.

Tabla 4. Porcentaje de aparición de léxico disponible y léxico disponible común a la variable externa sexo en informantes $\mathrm{NI}$ 


\begin{tabular}{llllll}
\hline $\begin{array}{l}\text { Noción } \\
\text { Específica }\end{array}$ & Nivel español & Ítem léxico & $\begin{array}{l}\text { N. } \\
\text { palabras }\end{array}$ & $\begin{array}{l}\text { Porcentaje } \\
\text { aparición }\end{array}$ & de \\
\hline 2 & A1 & Inteligente & 3 & $7,1 \%$ & $\mathrm{H}$ \\
\hline 2 & A2 & Agradable & 1 & $2,4 \%$ & $\mathrm{H}$ \\
\hline 2 & No inventariada & Feliz & 2 & $4,8 \%$ & $\mathrm{M}$ \\
\hline 2 & No inventariada & Bueno & 1 & $2,4 \%$ & $\mathrm{H}$ \\
\hline 4 & A1 & Familia & 6 & $14,2 \%$ & $\mathrm{M}$ \\
\hline 4 & A1 & Padres & 1 & $2,4 \%$ & $\mathrm{H}$ \\
\hline 4 & A1 & Padre & 1 & $2,4 \%$ & $\mathrm{M}$ \\
\hline 4 & A1 & Madre & 9 & $21,4 \%$ & $\mathrm{M} / \mathrm{H}$ \\
\hline 4 & A1 & Hijo (a) & 8 & $19 \%$ & $\mathrm{M} / \mathrm{H}$ \\
\hline 4 & A1 & Abuelo (a) & 3 & $7,1 \%$ & $\mathrm{M}$ \\
\hline 4 & A1 & Marido & 3 & $7,1 \%$ & $\mathrm{H}$ \\
\hline 4 & A2 & Mamá & 3 & $7,1 \%$ & $\mathrm{M}$ \\
\hline 4 & No inventariada (C1) & Esposo (a) & 1 & $2,4 \%$ & $\mathrm{H}$ \\
\hline 4 & No inventariada (B1) & Matrimonio & 1 & $2,4 \%$ & $\mathrm{H}$ \\
\hline Total & & & 43 & $100 \%$ & $14,2 \%$ \\
\hline
\end{tabular}

A partir del análisis del léxico disponible en los informantes de nivel intermedio de ambos sexos $(\mathrm{M} / \mathrm{H})$, al inicio del período de inmersión, corresponde a 2 ítems léxicos entre 14 aparecidos en uso en el corpus: 'Madre' con un 21,4 \% de aparición e 'Hijo' con un 19 \% de aparición, que en total corresponden al 14,2\% del léxico disponible total aparecido en uso en el corpus.

De esta manera, se puede concluir que, en principio, es posible delimitar un léxico en común para ambos sexos por nivel de adquisición; sin embargo, los datos correspondientes a los informantes del nivel intermedio no son representativos, debido a que como ya se ha recordado anteriormente, corresponden solo a ocho informantes, cuatro hombres y cuatro mujeres, pero sirven inicialmente, para poder obtener conclusiones preliminares y esbozar una posible tendencia. Los datos recogidos de los informantes de nivel avanzado podrían considerarse relativamente representativos, ya que permiten concluir, al menos de forma preliminar, que a partir de una prueba de diagnóstico como la que se les aplica, es posible dilucidar el léxico disponible real con el que cuentan, tanto separados por sexo, como en general, para así producir una posible herramienta pedagógica o generar una base desde la que se puede partir el aprendizaje del léxico disponible. 


\section{Conclusiones}

Existe una amplia asimilación del léxico comprendido en la categoría de sustantivos dentro de la Noción Específica 4 correspondiente a las 'Relaciones familiares', por sobre el léxico comprendido en la categoría de adjetivos y correspondiente en su mayoría a la Noción Específica 2, dentro de 'Carácter y personalidad'. En este caso, se pudo ver que las mujeres tienen mayor facilidad para asimilar y usar los adjetivos que los hombres, y que los hombres tienden a usar más veces los ítems léxicos de los que, eventualmente, se sienten seguros, priorizando la repetición por sobre la variedad, mientras las mujeres priorizan la variedad léxica, aun cuando contextualmente pueden no utilizar de la manera más adecuada el matiz semántico-gramatical del término elegido, lo que daría cuenta de una mayor seguridad lingüística. De esta manera, con un enfoque descriptivo, se pudo comprobar la primera hipótesis, referente a que existe una diferencia en el uso de léxico disponible al inicio de la inmersión entre hombres y mujeres que estudian español como segunda lengua.

El total de ítems léxicos en uso es de 42 términos, de los que 17 corresponden a léxico inventariado en las Nociones especificadas, más 3 ítems léxicos que pertenecen a estas Nociones, pero en diferente nivel de referencia, y 22 ítems léxicos de uso en contexto no inventariados. La repetición total de palabras es de 295 apariciones. A grandes rasgos, se puede señalar que los estudiantes de español como segunda lengua cuentan con un bajo nivel de asimilación del léxico disponible al inicio de la etapa de inmersión, sobre todo, en el inicio del nivel avanzado. Así, para poder comunicarse, tienden a repetir aquellas palabras que conocen y de cuyo uso se sienten seguros. De esta manera, la ejercitación del léxico contextual y de las variantes posibles de cada léxico con sus respectivos matices semánticos se hace indispensable desde el inicio de la inmersión.

Como conclusión final, cabe señalar que se pudo delimitar un léxico en común para ambos sexos, por nivel de adquisición, correspondiente al $37 \%$ en los informantes de nivel avanzado y $14,2 \%$ en los informantes de nivel intermedio, lo que permitió corroborar descriptivamente la segunda hipótesis: que se puede determinar un léxico común a ambos sexos al inicio de la inmersión en Santiago de Chile de los estudiante de español como segunda lengua. Este léxico común para ambos sexos encontrado a partir de una prueba de 
diagnóstico como la que se aplica, puede ser el punto de partida para desarrollar material de aprendizaje que sirva para reforzar la adquisición de léxico y su consecuente asimilación, y así contribuir a que el español sea aprendido desde la riqueza léxica que lo caracteriza, así como para reforzar el léxico que ya se encuentra interiorizado.

\section{Referencias bibliográficas}

Ávila, R. (1997). Variación léxica: connotación, denotación, autorregulación. Anuario de Letras, XXXL, 77-102.

Calsamiglia, H. y Tusón, A. (1999). Las cosas del decir. Manual de análisis del discurso. Barcelona: Ariel.

Centro Virtual Cervantes. (2015). Diccionario de términos clave de ELE. Competencia comunicativa. Recuperado de

http://cvc.cervantes.es/ensenanza/biblioteca_ele/diccio_ele/diccionario/competenciacomuni cativa.htm. Consultado el: el 5 de enero de 2017.

Coates, J. (2009). Mujeres, hombres y lenguaje. Un acercamiento sociolingüístico a las diferencias de género. México: Fondo de Cultura Económica.

Consejo de Europa. (2002). Marco Común Europeo de Referencia para la Enseñanza de Lenguas. Strasbourg: Council of Europe.

Escoriza, L. (2002). La variación lingüística. Propuesta de delimitación de variantes en el nivel léxico (Tesis doctoral). Cádiz: Universidad de Cádiz.

Escoriza, L. (2004). Posibilidades de aplicación del concepto de variación lingüística al nivel léxico en el ámbito de la sociolingüística. En: M. Villayandre (ed.), Actas del V Congreso de Lingüística General (3 vol.). Madrid: Arco/Libros.

Galdames, A., Toledo, G. y Guerrero, S. (m/i). Disponibilidad léxica de aprendientes de español como segunda lengua en contexto de inmersión: una plataforma para la enseñanza del léxico.

Gallego, D. (2014). “Léxico disponible de 82 inmigrantes estudiantes de español en la ciudad de Alcalá de Henares". En: Lengua y migración 6(2), 95-123.

Labov, W. (1966). The social stratification of English in New York city. Washington: Center for Applied Linguistics. 
López Morales, H. (1994). Métodos de investigación lingüística. Salamanca: Colegio de España.

López Morales, H. (2004). Sociolingüística. Madrid: Gredos.

López Morales, H. (2011). “Los índices de riqueza léxica y la enseñanza de lenguas”. En: J. Guervós, H. Bongaerts, J. Sánchez y M. Seseña (coords.), Del texto a la lengua: La aplicación de los textos a la enseñanza-aprendizaje del español L2-LE (vol. 1, 1528).

Morante, R. (2005). El desarrollo del conocimiento léxico en segundas lenguas. Madrid: Arco Libros.

Moreno Fernández, F. (2009). Principios de sociolingüística y sociología del español. Barcelona: Ariel.

Moreno Fernández, F. (2010). Las variedades de la lengua española y su enseñanza. Madrid: Arco Libros.

Moreno de Alba, J. (2006). “Unidad y diversidad del español: el léxico”. En: Nueva Revista de Filología Hispánica, LIV(1), 175-189.

Instituto Cervantes. (2007). Plan Curricular del Instituto Cervantes. Madrid: Instituto Cervantes, Biblioteca Nueva.

Trudgill, Peter. (1974). The social differentiation of English in Norwich. Cambridge: Cambridge University Press.

Sánchez, C. (2005). "Naturaleza gramatical del léxico disponible en informantes de español como lengua extranjera". En: Interlingüística 16(2), 977-986.

Serrano, M. J. (2011). Sociolingüística. Barcelona: Ediciones del Serbal.

Silva-Corvalán, C. (2001). Sociolingüistica y pragmática del español. Washington: Georgetown University Press. 


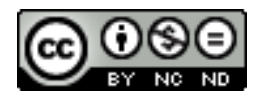

Esta obra está bajo una licencia de Creative Commons Reconocimiento-NoComercialSinObraDerivada 4.0 Internacional 